\title{
Presentation and Classification of Rare Primary Vasculitides in Children
}

\author{
Lythgoe Hanna ${ }^{1,2^{*}}$, Guratsky $\mathrm{V}^{3}$ and Baildam $\mathrm{E}^{3}$ \\ ${ }^{1}$ Department of Women's and Children's Health, Institute of Translational Medicine, University of Liverpool, UK \\ ${ }^{2}$ NIHR Alder Hey Clinical Research Facility, Alder Hey Children's NHS Foundation Trust, Liverpool, UK \\ ${ }^{3}$ Department of Paediatric Rheumatology, Alder Hey Children's NHS Foundation Trust, Liverpool, UK
}

"Corresponding author: Lythgoe Hanna, Department of Women's and Children's Health, Institute of Translational Medicine, University of Liverpool, UK, Tel: 0151228 4811; Fax: 0151228 0328; E-mail: hannalythgoe@doctors.org.uk

Received date: April 21, 2016; Accepted date: May 05, 2016; Published date: May 08, 2016

Copyright: (C) 2016 Lythgoe $\mathrm{H}$, et al. This is an open-access article distributed under the terms of the Creative Commons Attribution License, which permits unrestricted use, distribution, and reproduction in any medium, provided the original author and source are credited.

\begin{abstract}
Primary vasculitides, where blood vessels are the primary site of inflammation, with the exception of Henoch Schönlein purpura (HSP) and Kawasaki's disease (KD) are rare in childhood but when present, cause significant morbidity and mortality. This review provides an update on classification and presentation of rare vasculitides in children, through considering our experience in three cases in context with the current literature. We have demonstrated the challenges to diagnosis and how diagnosis can easily be missed if vasculitides are not carefully considered and looked for. The introduction of classification criteria has been important to allow more research into rare paediatric vasculitides but development of diagnostic criteria may be useful. Vasculitides should be considered in all patients presenting with multi-system features.
\end{abstract}

Keywords: Vasculitides; Ischaemia; Granulomatosis

\section{Introduction}

Primary vasculitis (where blood vessels are the primary site of inflammation), with the exception of Henoch-Schönlein purpura (HSP) and Kawasaki's disease (KD), are rare in childhood [1]. However, when they do present they are associated with significant morbidity and mortality and early diagnosis is important to ensure prompt treatment [2].

Diagnosis is based on a spectrum of clinical features and associated laboratory and histopathological findings. Presenting features are often insidious, non-specific and may mimic other diseases; diagnosis can therefore be challenging. Presentation can vary from benign, selflimiting cutaneous disease to severe, multi-system, life-threatening disease.

Symptoms of systemic inflammation may develop due to release of chemical mediators from inflamed blood vessels. Constitutional symptoms such as fever, malaise, arthritis/myalgia and raised inflammatory markers may be the first features of disease. However, systemic inflammation may not occur at all in those with localised disease.

More specific clinical features occur with involvement of individual organ systems. Inflammation of the vessel wall leads to increased vascular permeability and haemorrhage causing local symptoms such as purpuric rash with skin involvement or haemoptysis and breathlessness with pulmonary involvement. Narrowing or occlusion of affected vessels can lead to ischaemia or infarction causing local symptoms such as necrotic skin ulcers or mononeuritis multiplex.

This review focuses on primary vasculitides but it is important to be aware that vasculitis can occur in response to a number of other diseases such as infection and other connective tissue diseases. Infection must be considered and ruled out in all suspected cases of vasculitis as infection can mimic vasculitis and may also be the underlying cause. Immunosuppressant treatments may be commenced which could be dangerous if used in the context of unrecognised infection [3].

This review will aim to provide an update on classification and presentation of rare vasculitides in children through considering out experience in three cases in context with the current literature.

\section{Classification}

Vasculitides presenting in childhood have different aetiology, clinical presentations and prognosis to those presenting in adulthood [4]. It is therefore important to have classification criteria which are specific to children. New classification criteria were proposed by the vasculitis working group of the Paediatric Rheumatology European Society (PRES) in 2005. Classification is primarily based on the size of the predominantly affected vessels and whether it is granulomatous or not. An overview of these classification criteria is shown in (Box 1). With support from the European League Against Rheumatism (EULAR), the Paediatric Rheumatology International Trials Organization (PRINTO) and PRES, a formal statistical validation process with large-scale data collection was completed and finalised in the 2008 Ankara Consensus Conference6. There are now specific childhood classification criteria for granulomatosis with polyangiitis (GPA), Henoch-Schönlein Purpura (HSP), childhood polyarteritis nodosa (PAN) and Takayasu's arteritis (TA) [6].

These classification criteria are important to advance research in these rare diseases and are already in use in international clinical trials including the MYPAN trial (an open label randomised controlled trial of mycophenolate versus cyclophosphamide for the induction of remission of childhood polyarteritis nodosa) and the PePREs trial (a phase IIa, international, multicentre, open-label, uncontrolled study to evaluate the safety and pharmacokinetics of rituximab in patients with 
severe granulomatosis with polyangiitis or microscopic polyangiitis) $[7,8]$.

It is important to note that these are classification rather than diagnostic criteria and they may not capture all patients with the defined disease [9]. As such, when making a diagnosis it is important to consider all the clinical features, only some of which may be part of the classification criteria [10]. Diagnostic criteria for childhood vasculitis are not currently available.

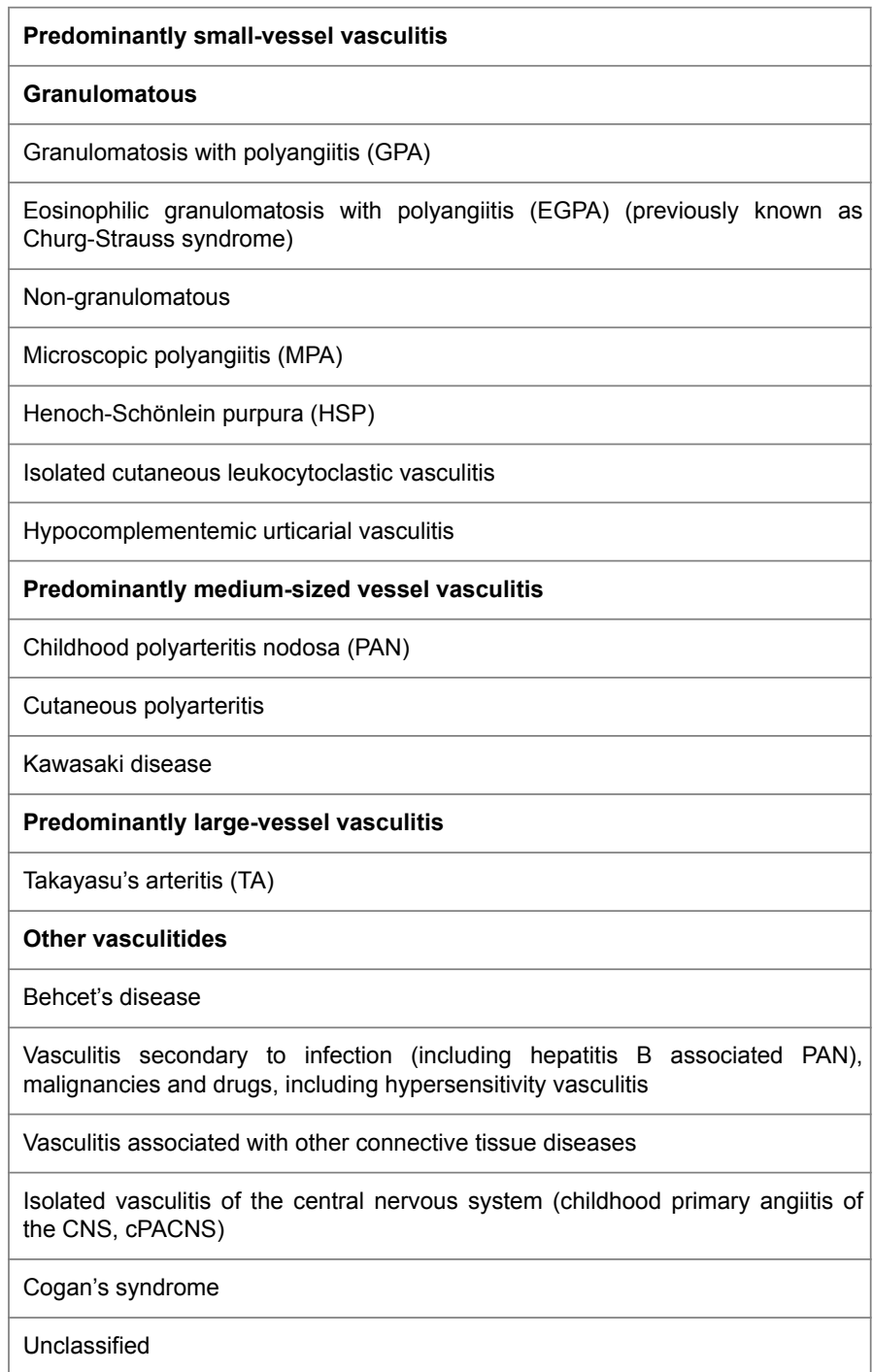

Box 1: Classification of childhood Vasculitis [5]

\section{Small-vessel vasculitis}

Small-vessel vasculitis is where disease predominantly affects the small blood vessels such as capillaries and venules. For example, involvement of cutaneous post-capillary venules causes palpable purpura; involvement of glomerular capillaries causes renal disease such as haematuria, proteinuria and renal failure and involvement of pulmonary capillaries causes pulmonary disease such as breathlessness, haemoptysis and widespread alveolar shadowing on chest X-ray [3].
Small-vessel vasculitis may be granulomatous (GPA, EGPA) or nongranulomatous (MPA, HSP, isolated cutaneous leukocytoclastic vasculitis or hypocomplementemic vasculitis). The ANCA-associated vasculitides (GPA, EGPA, MPA) may present with a severe, rapidly progressive course which may include pulmonary haemorrhage, respiratory failure and acute renal failure. They can also present with a more chronic course with mainly localised disease. These presentations may coexist or present sequentially in individual patients [11].

\section{GPA}

GPA (previously known as Wegener's granulomatosis), whilst still rare, is the most common of the ANCA-associated vasculitides in children and has a median age of presentation of $12-14$ years $[7,8]$. It can present with multiple symptoms and diagnosis is not always straight-forward (Figure 1). In a review of 65 children with GPA $89 \%$ of children presented with constitutional symptoms including fatigue/ malaise, fever or weight loss; $80 \%$ of children had ENT involvement including nasal involvement, sinusitis, stridor (from laryngeal or tracheal involvement) etc; $80 \%$ presented with pulmonary involvement such as shortness of breath, chronic cough, haemoptysis; $75 \%$ present with renal involvement with $11 \%$ of these requiring dialysis; $57 \%$ had musculoskeletal features; $41.5 \%$ gastrointestinal features; $35 \%$ cutaneous features and $89 \%$ of patients were cANCA or pANCA positive by immunofluorescence (Box 2) [12]. Constitutional and ENT features are more common in childhood-onset disease than adultonset disease [13]. Proptosis may also be a presenting feature. Classification criteria for GPA have been defined and are shown in (Box 3).

\begin{tabular}{|l|}
\hline Illustrative case 1 - a presentation of GPA \\
\hline 10 year old girl \\
\hline $\begin{array}{l}\text { Aged } 9 \text { years developed limping and reduced movement in her left hip. X- } \\
\text { rayand inflammatory markers were normal. Symptoms resolved spontaneously. }\end{array}$ \\
\hline $\begin{array}{l}\text { Aged } 10 \text { years developed nasal crusting, epistaxis and blocked nose. Nasal } \\
\text { endoscopy showed total nasal obstruction due to crusting. CT scan showed mild } \\
\text { mucosal thickening of the maxillary sinuses. Also diagnosed with left glue ear } \\
\text { with associated hearing loss. }\end{array}$ \\
\hline $\begin{array}{l}\text { Two months later she was referred to ophthalmology with red eye. She was } \\
\text { initially treated for possible staphylococcal sensitivity. She was later diagnosed } \\
\text { with bilateral episcleritis. }\end{array}$ \\
\hline $\begin{array}{l}\text { She then developed arthralgia with early morning stiffness, mouth ulcers and } \\
\text { mucus in her stools. She was admitted for further investigation. }\end{array}$ \\
\hline $\begin{array}{l}\text { During admission she developed an ulcer at the top of her finger and on her } \\
\text { bottom lip. } \\
\text { She underwent upper and lower gastrointestinal (GI) endoscopy which showed } \\
\text { multiple ulcers and a provisional diagnosis of Crohn's disease was made. She } \\
\text { was started on modular feeds and azathioprine. }\end{array}$ \\
\hline $\begin{array}{l}\text { Further results showed high cANCA levels against PR3. Histopathology from the } \\
\text { Gl biopsy was consistent with ANCA vasculitis. }\end{array}$ \\
\hline Saddle nose deformity was also present \\
\hline \begin{tabular}{l} 
Diagnosis of GPA was made and appropriate treatment commenced. \\
\hline
\end{tabular} \\
\hline
\end{tabular}

Box 2: Case study 1 
Page 3 of 5

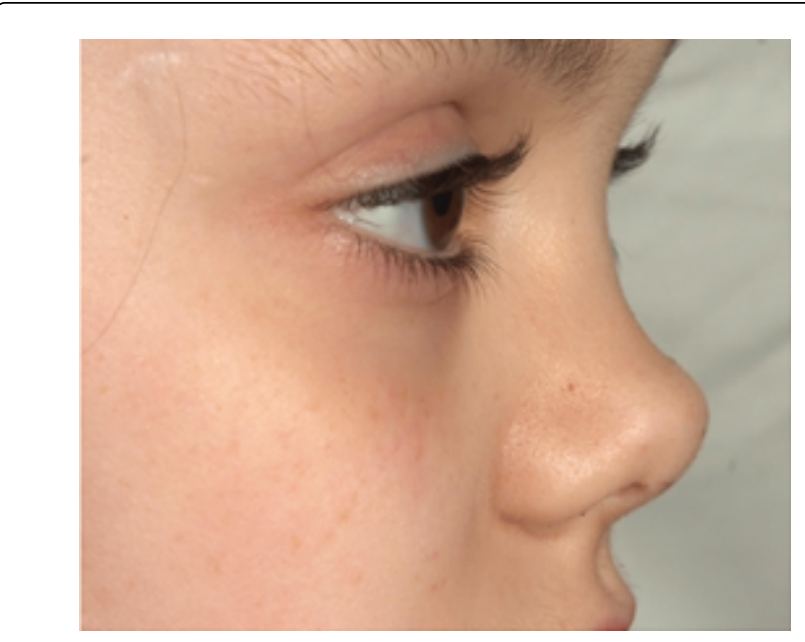

Figure 1: Case study 1

\section{Classification criteria for GPA}

Requires at least 3 of the following 6 criteria:

Histopathology (granulomatous inflammation within the wall of an artery or in the perivascular or extravascular area)

Upper airway involvement (chronic purulent or bloody nasal discharge or recurrent epistaxis/crusts/granulomata; chronic or recurrent sinus inflammation; nasal septum perforation or saddle nose deformity)

Laryngo-tracheo-bronchial involvement (subglottic, tracheal or bronchial stenoses)

Pulmonary involvement (chest X-ray or CT showing the presence of nodules, cavities or fixed infiltrates)

ANCA (ANCA positivity by immunofluorescence or by ELISA MRO ANCA or PR3 ANCA)

Renal involvement (proteinuria; haematuria or red blood cell casts; necrotising pauci-immune glomerulonephritis)

Box 3: EULAR/PRINTO/PRES classification for childhood GPA [6]

\section{EGPA}

EGPA usually presents with asthma and allergic rhinitis with an eosinophilia. The mean age of presentation in childhood is 12 years of age [14]. Other common features include pulmonary infiltrates (85\%), sinusitis (77\%), cardiac disease (55\%), skin involvement (66\%), gastrointestinal involvement (40\%) and peripheral nerve involvement (39\%) [9]. About $25 \%$ of children are ANCA positive [14]. Pulmonary infiltrates and cardiomyopathy were seen more commonly in children than in adults with worse mortality rates in children, recent review from a tertiary rheumatology service showed a mortality rate of $15 \%$ $[14,15]$.

\section{MPA}

MPA most commonly includes pulmonary capillaritis and necrotising glomerulonephritis [16]. A review of 7 paediatric patients found all patients had renal involvement (2 requiring dialysis), skin involvement (purpura) and constitutional features at diagnosis [17].
The clinical features of MPA are similar to PAN (see below) but the presence of rapidly progressive glomerulonephritis rules out PAN [17].

\section{Medium-sized vessel vasculitis}

Medium-sized vessel vasculitis occurs where disease primarily affects small and medium sized arteries. For example, involvement of small cutaneous arteries may cause necrotic lesions and ulcers; involvement of the mesenteric artery may cause abdominal pain, gastrointestinal bleeding and perforation and involvement of epineural arteries may cause mononeuritis multiplex (Box 4).

Illustrative case 2 - PAN

9 year old presented with purpuric rash primarily on lower limbs but also present on trunk and upper limbs. Associated with arthritis

Microscopic haematuria noted

HSP diagnosed

10 months later symptoms persisted and patient referred to rheumatology service

Additional symptoms noted including headache, fatigue, weight loss and abdominal pain

Investigations demonstrated raised ESR, positive ANA, and subtle changes in the kidneys consistent with vasculitis on angiogram

Patient recruited to MYPAN trial and treated with I.V. cyclophosphamide

Box 4: Case study 2

\section{PAN}

PAN is a necrotising vasculitis classically associated with aneurysmal nodules on the walls of medium-sized vessels although more non-specific vascular abnormalities are often found on angiogram. It is the most common form of systemic vasculitis after HSP and KD in children with average onset between 7-11 years and is more common in boys [10]. PAN classically presents with non-specific symptoms including malaise, fever, weight loss, skin rash, myalgia, abdominal pain and arthropathy [18]. Livedo reticularis is also a characteristic feature [10]. PAN may also present with ischaemic heart or testicular pain; renal manifestations including hypertension, haematuria and proteinuria and/or neurological features such as hemiplegia, visual loss and mononeuritis multiplex $[10,19,20]$. Cutaneous involvement is variable and may imitate HSP (see case study 2) or erythema multiforme. Classification criteria for PAN are shown in (Box 5).

\section{Classification criteria for PAN}

Histopathology (evidence of necrotising vasculitis in small or medium sized arteries) or angiographic abnormalities (angiography showing aneurysm, stenoses or occlusion of a medium or small sized vessel, not due to fibromuscular dysplasia, or other non-inflammatory causes) plus at least one of the following criteria:

Skin involvement (livedo reticularis, skin nodules, superficial skin infarctions or deep skin infarctions)

Myalgia or muscle tenderness

Hypertension (systolic/diastolic blood pressure >95th centile for height)

Peripheral neuropathy (sensory peripheral neuropathy or motor mononeuritis multiplex) 
Page 4 of 5

Renal involvement (proteinuria, haematuria or impaired renal function)

Box 5: EULAR/PRINTO/PRES classification for childhood PAN [6]

\section{Large-vessel vasculitis}

Large vessel vasculitis affects the aorta and its branches. Involvement of the thoracic aorta and its branches causes features such as limb claudication, absent pulses, unequal blood pressure and bruits [18]. Takayasu's arteritis is considered the only large vessel arteritis in children as giant cell arteritis rarely presents before the age of 50 years (Box 6) [21].

\begin{tabular}{|l|}
\hline Illustrative case 3 - Takayasu's arteritis \\
\hline 13 year old under rheumatology team for erosive arthropathy \\
\hline Presented with frontal headache and loss of vision on right side \\
\hline MRI brain reported as left occipital infarct with normal MRA/MRV \\
\hline Treated with aspirin \\
\hline $\begin{array}{l}\text { Further examination found absent right radial pulse, bruit over subclavian area } \\
\text { and unable to record BP on right arm }\end{array}$ \\
\hline $\begin{array}{l}\text { Doppler of right arm showed obstructive waveform in the radial and brachial } \\
\text { flows }\end{array}$ \\
\hline CT angiogram subsequently found narrowing of the right subclavian artery \\
\hline Raised ESR recorded \\
\hline Diagnosis of Takayasu's arteritis was made \\
\hline Treated with steroids and cyclophosphamide \\
\hline
\end{tabular}

Box 6: Case study 3

\section{Takayasu's arteritis}

Takayasu's arteritis most commonly presents in childhood with hypertension, heart failure or neurological symptoms [21]. Hypertension is the most frequent clinical feature at presentation in both adults and children and affects up to $93 \%$ of patients presenting in childhood [20,22]. The overall clinical manifestations of children affected by TA may differ from affected adults11. A review of 19 children with TA found the most common symptoms were headache (89\%), abdominal pain (37\%), claudication (32\%), fever $(26 \%)$ and weight loss (10\%) [20]. The most common clinical signs were hypertension (89\%), absent pulses (58\%) and bruits (42\%) [20]. Takayasu's arteritis, like other vasculitides, may be missed if not carefully looked for (see case study 3). Classification criteria for TA are shown in (Box 7).

\section{Classification criteria for TA}

Angiographic abnormality (angiography of the aorta or its main branches and pulmonary arteries showing aneurysm/dilatation, narrowing, occlusion or thickened arterial wall) plus one of the following:

Pulse deficit or claudication (lost/decreased/unequal peripheral artery pulse(s) or focal muscle pain induced by physical activity)

Blood pressure discrepancy (discrepancy of four limb systolic blood pressure > $10 \mathrm{mmHg}$ difference in any limb)
Bruits (audible murmurs or palpable thrills over large arteries)

Hypertension (systolic/diastolic blood pressure $>95$ th centile for height)

Acute phase reactant (Erythrocyte sedimentation rate $>20 \mathrm{~mm}$ per first hour or CRP any value above normal)

Box 7: EULAR/PRINTO/PRES classification for childhood Takayasu's arteritis [6]

\section{Investigations}

Patients with suspected vasculitis require a detailed history and examination. Investigations should then be carried out to look for radiological/histopathological proof of diagnosis; identify the subtype of vasculitis; determine the extent of the vasculitis; and rule out infection and other diagnoses. Histopathology is of utmost important in diagnosis and therefore examination should focus on trying to consider an appropriate area to biopsy.

Tissue diagnosis should be carried out wherever possible. The site of biopsy will depend on the site of presentation. Where there is evidence of multi-system involvement the biopsy site should be chosen according to its likelihood of affecting treatment decisions. However, tissue diagnosis is not always possible, for example in patients with large vessel vasculitis or those with medium vessel vasculitis with no accessible tissue and angiograms should be considered in these patients. Spiral computed tomographic angiography may aid rapid diagnosis in patients with suspected PAN [23,24]. Magnetic resonance angiogram of the thoracic aorta and aortic roots would be the investigation of choice for children with suspected TA [3,24].

Laboratory evaluation for vasculitis should include a full blood count and acute phase reactants including erythrocyte sedimentation rate and C-reactive protein. Depending on the presentation ANCAs, complements, cryoglobulin and IgE levels may help to determine the subtype of vasculitis. Tests to assess the extent of vasculitis including urine microscopy, renal function tests, liver function tests, chest X-ray, and echocardiogram and nerve conduction studies/electromyography should be considered. Additional tests may be required to rule out differential diagnoses such as blood cultures, hepatitis screen, HIV screen, ANA, anti-phospholipid antibodies.

\section{Discussion}

Although rare, it is likely that vasculitis is underdiagnosed in paediatrics due to lack of awareness. We have demonstrated through case illustrations the challenges to diagnosis and how diagnosis can easily be missed if vasculitides are not carefully considered and looked for.

Thorough medical history and examination should be carried out in all paediatric patients with possible features of multi-system disease in order to ensure that all features are identified. In our experience cases with missed or delayed diagnoses are often due to failure to be complete in small aspects of assessment. For example, a single missing or reduced pulse may be the only clue in a stroke patient with an underlying diagnosis of Takayasu's arteritis, so that failure to palpate all peripheral pulses may lead to a missed diagnosis. Unless blood pressure and urinalysis are performed routinely in new patients nephritis will be missed. ESR measurement may be raised with more normal CRP levels in the auto-immune conditions and will be missed where CRP levels are measured in isolation. Similarly eosinophil 
counts are often raised in all types of vasculitis but especially in EGPA. However, they are exquisitely sensitive to steroids used to treat asthma and the significance of the raised level for detection of the underlying diagnosis may be missed.

Particular red flag features include atypical asthma, new onset stridor or proptosis, and persistent ENT problems. Expert paediatric rheumatology opinion should be sought urgently where multi-system features exist.

It is important not to miss these conditions due to their high mortality rates without appropriate treatment. Early treatment is likely to improve outcome for children with vasculitis and may reverse existing features (see case study 3 ). Although paediatricians are always vigilant for malignancy and $\mathrm{TB}$ as occult serious disorders, it is imperative that the vasculitides are added to the list of disorders requiring constant awareness and consideration.

\section{Conclusion}

Clinical manifestations of primary paediatric vasculitides are extremely varied and diagnosis remains challenging. The introduction of classification criteria has been important to allow more research into rare paediatric vasculitides but development of diagnostic criteria may be useful. Vasculitides should be considered in all patients presenting with multi-system features.

\section{References}

1. Gardner-Medwin JM, Dolezalova P, Cummins C, Southwood TR (2002) Incidence of Henoch-Schönlein purpura, Kawasaki disease, and rare vasculitides in children of different ethnic origins. Lancet 360: 1197-1202.

2. Dillon MJ (2006) Vasculitis treatment - new therapeutic approaches. Eur J Pediatr 165: 351-357.

3. Suresh E (2006) Diagnostic approach to patients with suspected vasculitis. Postgrad Med J 82: 483-488.

4. Tullus K, Marks SD (2009) Vasculitis in children and adolescents: clinical presentation, etiopathogenesis, and treatment. Paediatr Drugs 11 375-380.

5. Ozen S, Ruperto N, Dillon MJ, Bagga A, Barron K, et al. (2006) EULAR/ PReS endorsed consensus criteria for the classification of childhood vasculitides. Ann Rheum Dis 65: 936-941.

6. Ozen S, Pistorio A, Iusan SM, Bakkaloglu A, Herlin T, et al. (2010) EULAR/PRINTO/PRES criteria for Henoch-Schönlein purpura childhood polyarteritis nodosa, childhood Wegener granulomatosis and childhood Takayasu arteritis: Ankara 2008. Part II: Final classification criteria. Ann Rheum Dis 69: 798-806.

7. Granville H (2015) MYPAN Trial Summary.
8. Hoffman-La RocheA (2016) Study of Intravenous MabThera/Rituxan in Pediatric Patients with Severe Granulomatosis with Polyangiitis (Wegener's) or Microscopic Polyangiitis NCT01750697.

9. Aggarwal R, Ringold S, Khanna D, Neogi T, Johnson SR, et al. (2015) Distinctions between diagnostic and classification criteria? Arthritis Care Res (Hoboken) 67: 891-897.

10. Dillon MJ, Eleftheriou D, Brogan PA (2010) Medium-size-vessel vasculitis. Pediatr Nephrol 25: 1641-1652.

11. Eleftheriou D, Brogan PA (2009) Vasculitis in children. Best Pract Res Clin Rheumatol 23: 309-323

12. Cabral DA, Uribe AG, Benseler S, O'Neil KM, Hashkes PJ, et al. (2009) Classification, presentation, and initial treatment of Wegener's granulomatosis in childhood. Arthritis Rheum 60: 3413-3424.

13. Bohm M, Gonzalez Fernandez MI, Ozen S, Pistorio A, Dolezalova P, et al. (2014) Clinical features of childhood granulomatosis with polyangiitis (wegener's granulomatosis). Pediatr Rheumatol Online J 12: 18.

14. Zwerina J, Eger G, Englbrecht M, Manger B, Schett G, et al. (2009) Churg-Strauss Syndrome in Childhood: A Systematic Literature Review and Clinical Comparison with Adult Patients. Semin Arthritis Rheum 39: 108-115.

15. Eleftheriou D, Gale H, Pilkington C, Fenton M, Sebire NJ, et al. (2016) Eosinophilic granulomatosis with polyangiitis in childhood: retrospective experience from a tertiary referral centre in the UK. Rheumatology (Oxford) .

16. Jennette JC, Thomas DB, Falk RJ (2001) Microscopic polyangiitis (microscopic polyarteritis). Semin Diagn Pathol 18: 3-13

17. Peco-Antic A, Bonaci-Nikolic B, Basta-Jovanovic G, Kostic M, MarkovicLipkovski, et al. (2006) Childhood microscopic polyangiitis associated with MPO-ANCA. Pediatr Nephrol 21: 46-53.

18. Ozen S, Besbas N, Saatci U, Bakkaloglu A (1992) Diagnostic criteria for polyarteritis nodosa in childhood. J Pediatr 120: 206-209.

19. Maeda M, Kobayashi M, Okamoto S, Fuse T, Matsuyama T, et al. (1997) Clinical observation of 14 cases of childhood polyarteritis nodosa in Japan. Acta Paediatr Jpn 39: 277-279.

20. Cakar N, Ozçakar ZB, Soy D, Uçar Y, Fitöz S, et al. (2008) Renal involvement in childhood vasculitis. Nephron Clin Pract 108: c202-206.

21. Gulati A, Bagga A (2010) Large vessel vasculitis. Pediatr Nephrol 25: 1037-1048.

22. Hong CY, Yun YS, Choi JY, Su JH, Lee KS, et al. (1992) Takayasu arteritis in Korean children: clinical report of seventy cases. Heart Vessels Suppl 7: 91-96.

23. Ozçakar ZB, Yalçinkaya F, Fitoz S, Yüksel S, Acar B, et al. (2006) Polyarteritis nodosa: successful diagnostic imaging utilizing pulsed and color Doppler ultrasonography and computed tomography angiography. Eur J Pediatr 165: 120-123.

24. Stanson AW, Friese JL, Johnson CM, McKusick MA, Breen JF, et al. (2001) Polyarteritis nodosa: spectrum of angiographic findings. Radiographics 21: $151-159$ 